\title{
Physical Mechanics, a New Field in Engineering Science
}

\author{
H. S. TSIEN
}

Daniel and Florence Guggenheim Jet Propulsion Center, California Institute of Technology, Pasadena, Calif.

The purpose of physical mechanies is to predict the engineering behavior of matter in bulk from the microscopic properties of its molecular and atomic constituents. The constants and basic concepts of this new engineering science, of particular importance to rocket and jet propulsion, are discussed in this paper.

$\mathbf{T}$ HE term physical mechanics has been used in the past to indicate a course in classical mechanics for sophomores and juniors in college (1). ${ }^{2}$ Physical mechanics here is used to designate a new field in engineering science, the purpose of which is to predict the macroscopic behavior of matter, of interest to engineers, from the known microscopic properties of the constituents of matter. The need for such a branch of science arises originally from the advanced engineering problems in jet propulsion, aeronautics, and atomic power, but impact of this new discipline is inevitable on all fields of engineering. This article will discuss the contents of physical mechanics and its point of view. Above all, the purpose here is to attract the attention of scientists and engineers to this new and fruitful field.

\section{Basic Concepts}

The constituents of matter are molecules and atoms. The atom, in turn, consists of a central nucleus and an electron' cloud surrounding the nucleus. According to the prevailing view, the nucleus is ultimately made up of protons and neutrons. This relentless drive of physicist to the "heart" of the matter was perhaps motivated by the desire to interpret all nature phenomena by a unified theory from the elementary structures. During this development of physical science in the past century, there was a continuous interplay between two phases of the study. One phase was the investigation of the structure of molecules and atoms by analytical procedures such as $\mathrm{x}$-ray analysis and electron diffraction, molecular and atomic spectroscopy. The other phase was the explanation of the bulk properties of matter such as heat capacity, the pressure of fluids, from the molecular and atomic structure. The second phase of the investigation, developed by the use of the statistical mechanics and the kinetic theory, was of particular importance to the physicist and the chemists in that the earlier pictures of the molecular and atomic structures were quite uncertain and vague. Therefore the physical scientists needed the comfort of seeing their theory verified by "every-day" experience with matter in bulk.

The present knowledge of the molecular and atomic structures is, however, very complete and well founded. To the physical scientists then, the interpretation of the macroscopic behavior of matter from the atomic theory is only of side interest. A physicist's main interest is one step deeper: The structure of atomic nuclei and the properties of their constituent elementary particles. This situation in physics and chemistry leads naturally to a reversal of the procedure.

\footnotetext{
Received September 12, 1952

${ }^{1}$ Robert H. Goddard Professor of Jet Propulsion. The author is indebted to Dr. S. S. Penner, Assistant Professor of Jet Propulsion, for many stimulating discussions during the formulation of concepts in this paper.

${ }^{2}$ Numbers in parentheses refer to the References on page 16.
}

The statistical mechanics and the kinetic theory are not used to verify the atomic theory through the bulk properties of matter, but rather should be used to predict the bulk behaviors of matter from the known properties of the molecules and atoms. Since engineers always deal with matter in bulk, the prediction of bulk properties is then of vital interest to engineers, and is logically a branch of engineering science.

However, it might be argued that the mere fact of the engineer's interest in bulk properties of matter does not necessarily mean the need for physical mechanics. The bulk properties can be measured directly; then the theoretical calculations of physical mechanics will not be needed. This has been the situation till very recently. When the engineer needs the thermodynamic properties of steam or ammonia, he measures them. When the engineer needs the viscosity of water, he again measures it. If such direct measurements can be made easily with the required accuracy, there is no incentive to introduce new methods. Recently, however, particularly with the advent of rocket and jet propulsion engineering and nuclear engineering, bulk properties of matter at unusual conditions are required. For instance, thermodynamic properties at very high temperatures, say, $4000^{\circ} \mathrm{K}$, enter into engineering calculations. The experimental measurement of thermodynamic properties at such temperatures is certainly very difficult, if not impossible. On the other hand, the thermodynamic properties of gases at high temperatures can be calculated by the method of statistical mechanics with ease and certainty, once the properties of the constituent molecules and atoms are known. This circumstance can be easily understood by the observation that although the bulk temperature of the gas may be very high in the conventional engineering sense, the average energy of a single molecule or atom is quite moderate and within the range of certain knowledge of a physicist or a chemist. For instance, the average or representative kinetic energy of molecule or atom at a temperature $T$ in the absolute scale is $3 / 2 k T, k$ being Boltzmann's constant. The value of $k$ is given by

$$
\begin{aligned}
k & =1.380 \times 10^{-16} \mathrm{erg} \text { per }{ }^{\circ} \mathrm{K} \\
& =0.861 \times 10^{-4} \text { electron-volt per }{ }^{\circ} \mathrm{K}
\end{aligned}
$$

Therefore even at the "fantastic" temperature of $10,000^{\circ} \mathrm{K}$, the average kinetic energy of the atoms is still only 1.292 electron-volt, an energy at which the behavior of atoms is known with accuracy.

\section{Physical Mechanics as an Engineering Science}

The problems in physical mechanics can be classified into two categories: Problems in thermodynamic properties of matter at equilibrium, and problems in transport properties of matter not at equilibrium. Although the powerful methods of statistical mechanics are equally as applicable to matter at thermodynamic equilibrium as to matter not at equilibrium, concrete useful results are obtained easily only for the first case. For the transport properties, such as viscosity, heat conduction, and diffusion, the methods of the kinetic theory of matter give quantitative answers.

Examples of the first category of problems are the thermodynamic functions of gases, solids and liquids, the equation of states for imperfect or highly compressed gases, chemical equilibrium constants, and the thermodynamic behavior of 
electrically charged gases, such as gas containing ions and electrons, The previous investigations and results in this field are summarized, for example, in the two excellent books by Sir R. Fowler (2) and by Fowler and E. A. Guggenheim (3).

Examples of the second category of problems are, besides those already mentioned, the neutron diffusion in a nuclear reactor, and the moderation of fast neutrons from the fission nuclei to thermal neutrons by the reactor materials. Therefore many problems in nuclear engineering dealing with the macroscopic effects of elementary nuclear processes belong logically: to the science of physical mechanics. The theory of transport properties of gases is given in the famous book by S. Chapman and T. G. Cowling (4). Besides those problems of transport properties, the radiative emissivity of gases at high temperatures and the spectra obtained from regions of active combustion are also important problems of the second category.

It is evident that there has been certainly no lack of effort by physicists and physical chemists in the general field of what is here called physical mechanics. Physical chemists were particularly diligent in carrying out many extremely tedious calculations (5). Then, is the task of establishing physical mechanics as an engineering science that of simply disseminating this work of physicists and chemists among the engineering profession? Unfortunately, the task is not as simple as that. To establish physical mechanics as an engineering science, one must follow the example of other branches of engineering science, such as fluid mechanics, by introducing the successful guiding principles of the socalled "angewandte Mechanik." These principles of engineering science were first formulated and applied by a group of brilliant "applied mechanicists" of Göttingen University at the turn of this century (6). The following sections will be devoted to a discussion of these principles as applied to physical mechanics.

\section{Use of Approximate Models}

One of the principles of engineering science is the approximate solution of complex problem by using a simplified model which gives a satisfactory representation of reality. Because of the very fact that the model is simple, it cannot have all the properties of the real system, and it can be only designed to emphasize the most important feature of the real system under the particular physical situation concerned. Under a different physical situation, the real system may very well reveal a different property which is important. Then a different model has to be adopted. Therefore the success of the choice of models rests on the clear understanding of the physical circumstances. A "physical mechanicist" can have, however, assistance in this difficult task in two ways. He can always study the experimental observations connected with the phenomenon and thus gain insight to the problem. Then he is helped by knowing the logical requirement, that the models representing the same physical system, although they may be different under different situations, should nevertheless be compatible with each other and should not be contradictory to each other. The following is an example for the point under discussion.

From diffraction experiments and spectroscopic studies, the structure of molecules can be determined in terms of interatomic distances and bond angles. Such data, together with the van der Waals sizes of atoms, then give a definite model of a molecule as a structure of spheres (atoms) properly fused together. At ordinary temperatures, the rotation of molecules in a gas is fully excited. Thus the odd-shaped molecule is really under rapid rotation, and for molecules that are not excessively elongated the angular asymmetry is averaged out. Therefore, when one is considering the interaction of molecules, as, for example, in the calculation of the second virial coefficient and the transport properties of gases, one may well consider the molecules as spheres with a diameter $r^{*}$ equal to twice the maximum distance between the center of mass of the molecule and the boundary of the molecular model. This is shown to be actually the case by S. D. Hamann (7) and by Hamann and J. F. Pearse (8) for nonpolar molecules with the Lennard-Jones potential given by

$$
\epsilon(r)=\epsilon^{*}\left[\left(\frac{r^{*}}{r}\right)^{12}-\left(\frac{r^{*}}{r}\right)^{6}\right]
$$

where $r$ is the distance between the centers of mass of the molecules, $\epsilon$ the interaction energy, and $\epsilon^{*}$ the interaction energy at the distance $r^{*}$, the equilibrium distance. This is a very satisfactory state of affairs, as the chosen model of molecular interaction is entirely compatible with a wide range of other physical phenomena and concepts.

For polar molecules with permanent dipole moment $\mu, W$. H. Stockmayer (9) proposed an interaction potential which is a hybrid between the Lennard-Jones potential of Equation [1] and the dipole interaction potential,

$$
\begin{array}{r}
\epsilon=\epsilon^{*}\left[\left(\frac{r^{*}}{r}\right)^{12}-\left(\frac{r^{*}}{r}\right)^{6}\right]-\frac{\mu^{2}}{r^{3}}\left[2 \cos \theta_{1} \cos \theta_{2}-\right. \\
\left.\sin \theta_{1} \sin \theta_{2} \cos \varphi\right] .
\end{array}
$$

where $\theta_{1}, \theta_{2}$, and $\varphi$ are angles specifying the orientation of the interacting dipole axes. Following the work of W. H. Keesom $(10)$, Stockmayer calculated the second virial coefficient $B(T)$, where $T$ is the temperature, as

$$
\begin{aligned}
B(T)=\frac{1}{4} N \int_{0}^{\infty} r^{2} d r \int_{0}^{\pi} \sin \theta_{1} d \theta_{1} & \int_{0}^{\pi} \sin \theta_{2} d \theta_{2} \times \\
& \int_{0}^{2 \pi}\left[1-e^{-\epsilon / k T}\right] d \varphi .
\end{aligned}
$$

where $N$ is Avogadro's number, $\epsilon$ is the interaction potential given by Equation [2], and $k$ is Boltzmann's constant. The fitting of the experimentally determined second virial coefficients to Stockmayer's formula was carried out by J. O. Hirschfelder, F. T. McClure, and I. F. Weeks (11), and also by Hamann and Pearse (8) for methyl chloride and methyl fluoride. The results are, however, anomalous. For instance, the "size" $r$ " for steam and ammonia turns out to be approximately the van der Waals diameter of the oxygen atom and nitrogen atom, respectively, alone, without any room allowed for the hydrogen atoms in these molecules. It thus seems that Stockmayer's model of polar molecules is not entirely unquestionable.

An explanation of this difficulty can be obtained by observing the fact that if the molecule is under almost free rotation, then the dipole attraction and the dipole repulsion at any distance $r$ between molecules average out by the prevailing random orientation. Consequently, when the rotation of molecules is fully excited, the dipole moment of the molecule makes no contribution to the interaction potential. In other words, as far as the calculations of the second virial coeffici int and transport properties are concerned, it may be more realistic to neglect the difference between a polar molecule and a nonpolar molecule. This assumption will give a great simplification for the calculation of transport properties of polar gases. By fitting the second virial coefficient data for steam and ammonia to the formula for nonpolar molecules, satisfactory sizes $r^{*}$ for these molecules are obtained. Of course, the validity of this point of view has yet to be proved by a critical examination of the theory of virial coefficients.

\section{A Question of Methodology}

In recent years, perhaps because of the influx of mathematicians to the field of applied mechanics, the level of logical organization and of mathematical argument of research papers in this field is generally quite high. This modern trend, by itself, contributes to the elegance of presentation and facilitates the general understanding of the work. However, 
clarity of thought and the use of advanced mathematical tools have more than just this to contribute. In many problems of engineering science, the very complexity of the problems demands the most efficient and powerful tools for their solution. It is even to be expected that in many cases the problem will not be solved by any means other than the most efficient and powerful method. Therefore, to establish physical mechanics as an engineering science, it is necessary to emphasize this point of methodology.

As an example of lack of clarity of organization, one may take a recent paper (12) on the thermodynamic properties of completely ionized hydrogen. The criticism here is not that the results are incorrect, but rather that the same results could be obtained very simply and logically in a direct way. For this problem, one should first recognize that all thermodynamic properties of matter are contained in the partition function, or alternately, the free energy expression. Therefore the logical first step would be to establish the free energy of such ionized hydrogen, composed of equal numbers of positively charged protons and negatively charged electrons. But then the problem is exactly the same as the problem of solutions of electrolytes which dissociate into positive and negative ions. For solutions of electrolytes, there is the wellknown Debye-Hückel theory. ${ }^{3}$ The approximations involved and the validity of the assumptions involved in this theory are now clearly understood. By applying the Debye-Hückel theory to the problem of ionized hydrogen, one is at once clear about the power and the limitations of the solution. This alone is a worth-while saving in effort.

To show how easily the thermodynamics of completely ionized hydrogen can be determined with the rational method, one notes that if $F$ is the free energy of the assembly, $E$ the internal energy, $V$ the volume, $P$ the pressure, and $T$ the temperature, then according to the general laws of thermodynamics

$$
\begin{gathered}
P=-\frac{\partial F}{\partial V} \ldots \\
E=-T^{2} \frac{\partial(F / T)}{\partial T} .
\end{gathered}
$$

According to the Debye-Hückel theory, ${ }^{3}$ the first approximation to the free energy of Coulomb interaction $F^{e l}$ of equal numbers of positively and negatively charged particles with $\pm z e$ charge on each particle is

$$
F^{e l}=-\frac{2}{3} \sqrt{\pi} N k T\left(\frac{N^{1 / 3} z^{2} e^{2}}{V^{1 / 3} k T}\right)^{3 / 2}
$$

where $N$ is the total number of particles. Therefore, with Equations [4] and [5], the deviation $\Delta P$ of pressure and the deviation $\Delta E$ of internal energy from an assembly without Coulomb interaction are

$$
\begin{gathered}
\Delta P=-\frac{1}{3} \sqrt{\pi} \frac{N k T}{V}\left(\frac{N^{1 / 3} z^{2} e^{2}}{V^{1 / 3} k T}\right)^{8 / 2} \ldots \ldots \ldots[7] \\
\Delta E=-\sqrt{\pi} N k T\left(\frac{N^{1 / 3} z^{2} e^{2}}{V^{1 / 3} k T}\right)^{2 / 2} \ldots \ldots \ldots \ldots[8]
\end{gathered}
$$

For ionized hydrogen, $z=1$, and if $H$ is the atomic weight of hydrogen, and $\rho$ the density of the mixture

$$
N / V=2 \rho / H \ldots
$$

Therefore for ionized hydrogen

$$
\Delta P=-\frac{\sqrt{\pi}}{3}\left(\frac{2 \rho}{H}\right)^{s / 2} \frac{e^{3}}{\sqrt{k T}} \ldots
$$

and

$$
\Delta E=-\sqrt{\pi}\left(\frac{2 \rho}{H}\right)^{3 / 2} \frac{V e^{3}}{\sqrt{k T}}
$$

${ }^{3}$ See, for instance, Ref. 3, Chapter IX.
These results are the same as those in Reference 12 and are obtained here with very little calculation.

As an example of the advantages of mathematical technique in shortening the calculation, one may consider the second virial coefficient $B(T)$ for the Lennard-Jones potential of Equation [1]. It is known ${ }^{4}$ that, with $N$ as Avogardo's number, and

$$
\begin{aligned}
r_{0} & =2^{-1 / 6} r \ldots \ldots \ldots \ldots \ldots \ldots \ldots \\
\frac{B(T)}{2 \pi} N r_{0}^{3} & =-\frac{1}{4}\left(\frac{4 \epsilon^{*}}{k T}\right)^{1 / 4} \sum_{n=0}^{\infty} \frac{\Gamma\left(\frac{n}{2}-\frac{1}{4}\right)}{n !}\left(\frac{4 \epsilon^{*}}{k T}\right)^{n / 2} \ldots
\end{aligned}
$$

This series, although convergent for all values of $T$, is nevertheless inconvenient for very small values of temperature. For instance, it is stated (5) that for $k T / \epsilon^{*}=0.3$, about thirty terms are necessary to obtain an accuracy of five significant figures. The situation evidently calls for asymptotic expansion of the function $B(T)$ instead of the Taylor series of Equation [13]. The asymptotic series can be obtained easily as

$$
\begin{aligned}
& \frac{B(T)}{\frac{2 \pi}{3} N r_{0}{ }^{3}} \sim-\sqrt{\frac{\pi k T}{2 \epsilon^{*}}} e^{\epsilon^{*} / k T} \times \\
& \qquad \sum_{n=0}^{\infty} \frac{\Gamma\left(n+\frac{3}{4}\right) \Gamma\left(n+\frac{5}{4}\right)}{\Gamma\left(\frac{3}{4}\right) \Gamma\left(\frac{5}{4}\right) n !}\left(\frac{k T}{\epsilon^{*}}\right)^{n} \ldots[14]
\end{aligned}
$$

This expression has not only the advantage of being easier to use at small values of $T$, but also the advantage of clearly demonstrating the exponential behavior of the function at low temperatures. Such definite indication of functional behavior is often a help in understanding the interactions of the different elements of a problem.

\section{Concluding Remarks}

In the preceding discussion, the subject matter and the basic concepts of the new engineering science, physical mechanies, are outlined. It is an engineering science mainly because its foremost purpose is to help solve the engineering problems. And since it is an engineering science, physical mechanies should be a subject of training for any research or development engineers, of equal importance with fluid mechanics and solid mechanics. Because of its close relation to jet propulsion and rocket development, physical mechanies is now taught as a graduate course at the California Institute of Technology in the Daniel and Florence Guggenheim Jet Propulsion Center. However, the course is open to students of other fields of engineering with the proper preparation in mathematics, physics, and chemistry.

To skeptical purists among physicists and physical chemists, this discussion may appear to be overly optimistic or even immodest. For them, the author can only point to the unquestionable success of fluid mechanics and solid mechanics in modern engineering. There is no reason to expect the future of physical mechanics to be radically different.

\section{References}

1 See, for instance, "Physical Mechanies-An Intermediate Test for Students of the Physical Sciences," by R. B. Lindsay, D. Van Nostrand Co., New York, 1950.

2 "Statistical Mechanics," by R. H. Fowler, Cambridge University Press, 1936.

3 "Statistical Thermodynamics," by R. H. Fowler and E. A. Guggenheim, Cambridge University Press, 1949.

\footnotetext{
${ }^{4}$ See, for instance, Ref. 3, p. 280.
}

(Continued on page 35) 


\title{
Physical Mechanics, a New Field in Engineering Science
}

\author{
(Continued from page 16)
}

4 "The Mathematical Theory of Non-Uniform Gases," by S. Chapman and T. G. Cowling, Cambridge University Press, 1939.

5 See, for instance, the forthcoming book, "Properties of Gases," by J. O. Hirsehfelder, C. F. Curtiss, R. B. Bird, and E. L. Spotz. The manuscript of this book was issued by the authors as reports of the Naval Research Laboratory, Department of Chemistry, University of Wisconsin.

6 For this interesting anecdote of the birth of modern engineering science, see the chapter on "Aeronautics" by C. B. Millikan, in "Physics in Industry," American Institute of Physics, 1937.

7 "The Interpretation of Intermolecular Force Constants," by S. D. Hamann, Journal of Chemical Physics, vol, 19, 1951, p. 655.

8 "The Seeond Virial Coefficients of Some Organic Molecules," by S. D. Hamann and J. F. Pearse, Transactions of the Faraday Society, vol. 48 (2), 1952, pp. 101-106.

9 "Second Virial Coefficients of Polar Gases," by W. H. Stockmayer, Journal of Chemical Physics, vol. 9, 1941, pp. 398402.

10 "On the Deduction from Boltzmann's Entropy Principle of the Second Virial Coefficient"' by W. H. Keesom, Comm. Phys. Lab. Leiden, Suppl. 24B, §6, 1912.

11 "Second Virial Coefficients and the Force Between Complex Molecules," by J. O. Hirschfelder, F. T. McClure, and I. F. Weeks, Journal of Chemical Physics, vol. 10, 1942, pp. 201-211.

12 "On the Equation of State of Ionized Hydrogen," by R. E. Williamson, Astrophysics Journal, vol, 103, 1946, p. 139. 